\title{
CONTAMINANTES PRIORITARIOS EN TRUCHAS ARCOIRIS (Oncorhynchus mykiss) DEL MUNICIPIO DE MUTISCUA, NORTE DE SANTANDER, COLOMBIA
}

\section{PRIORITY CONTAMINANTS IN RAINBOW TROUTS (Oncorhynchus mykiss) FROM THE TOWNSHIP OF MUTISCUA, NORTE DE SANTANDER, COLOMBIA}

\author{
Alfonso Quijano ${ }^{1 *}$, Javier Navia², Maghdiel C. Portilla ${ }^{3}$ \\ Recibido para publicación: Diciembre 7 de 2013 - Aceptado para publicación: Abril 24 de 2014
}

\begin{abstract}
RESUMEN
Los Hidrocarburos Aromáticos Policíclicos (HAP) son compuestos importantes en la determinación de la contaminación atmosférica, producida por la combustión de fuentes móviles, contaminación industrial y la industria petrolera. Los HAP son considerados contaminantes prioritarios por La Agencia de Protección Ambiental de EE.UU (USEPA), debido a sus propiedades carcinogénicas y mutagénicas. Los HAP presentes en los alimentos, representan un riesgo potencial a los consumidores, se asocian fácilmente con la materia particulada y finalmente se depositan en el sedimento y en el agua. En los ecosistemas acuáticos los HAP se incorporan a la biota del entorno y los peces resultan excelentes biomarcadores de su presencia. La región de Mutiscua está influenciada por la contaminación atmosférica producida por las fuentes móviles a gasolina y diésel que circulan por una vía nacional aledaña al municipio. El creciente interés existente en la actualidad por la investigación en el campo de los compuestos orgánicos como los Contaminantes Prioritarios (CP), se debe a la identificación de efectos adversos de estos contaminantes sobre la salud y los ecosistemas. La presente investigación, es una evaluación de la presencia de los CP, en músculo y piel de trucha arco iris. La determinación de los HAP (CP) se realizó por Cromatografía de gases utilizando detector FID (Flamelonization Detector). Se logró identificar la presencia de una mezcla de Benzofluorantenos, considerados como posibles carcinógenos en humanos y a nivel de trazas la posible presencia del Benzo(a) pireno, también clasificado como carcinógeno.
\end{abstract}

Palabras Clave: cromatografía de gases, benzo(a)pireno, benzofluorantenos, Oncorhynchus mykiss, peces.

\begin{abstract}
Polycyclic Aromatic Hydrocarbons (PAHs) are important compounds in determining atmospheric pollution produced by combustion from mobile sources, industrial pollution and oil industry. PAHs are considered

\footnotetext{
${ }^{1 *}$ Ph.D. Química. Profesor Asociado. Director Laboratorio Control de Calidad. Facultad de Ciencias Básicas. Universidad de Pamplona, Colombia. Km 1 vía Bucaramanga, sector el Buque. cel 3166949582, fax 5685303 x 156. alfonsoquijanoparra@gmail.com

${ }^{2}$ Microbiólogo. Investigador Maestría en Alimentos. Facultad de Ingenierías. Departamento de Alimentos. Universidad de Pamplona, Colombia

${ }^{3}$ Ingeniera Alimentos. MsC en Ciencia y Tecnología de Alimentos. Facultad de Ingenierías. Departamento de Alimentos. Universidad de Pamplona, Colombia
} 
priority pollutants by the US Environmental Protection Agency US (USEPA), due to its carcinogenic and mutagenic properties. PAHs in food, represent a potential risk to consumers, are easily associated with the particulate matter and finally settle into the sediment and water. In aquatic ecosystems PAHs join the biota of the environment and the fish are excellent biomarkers of his presence. Mutiscua region is influenced by atmospheric pollution from mobile to petrol and diesel driven on a nearby national road to the municipality. The growing interest today in research in the field of organic compounds such as Priority Pollutants (PP), is due to the identification of adverse effects of these pollutants on health and ecosystems. This research is an assessment of the presence of PP in muscle and skin of rainbow trout. The determination of PAHs (PP) was performed by gas chromatography using FID (Flamelonization Detector). It was possible to identify the presence of a mixture of Benzofluorantenos considered as possible carcinogenic to humans, and trace level the possible presence of Benzo(a) pyrene, also classified as a carcinogen.

Key words: gas chromatography, benzo(a) pyrene, benzofluorantenos, Oncorhynchus mykiss, fish.

\section{INTRODUCCIÓN}

Los hidrocarburos aromáticos policíclicos (HAP) son un grupo de carcinógenos ambientales ampliamente distribuidos en el aire, en el suelo y en los alimentos. Los HAP se han convertido en contaminantes ambientales generalizados debido a su aparición en el petróleo, el carbón, el hollín, los contaminantes del aire y los aceites de corte (Hardin et al. 1992; Mudzinski 1993). Los HAP se encuentran en los gases de los vehículos, en el humo de la madera, en el humo del cigarrillo y también en altas concentraciones en los alimentos a la plancha y parrilla (IARC 1983; ATSDR 1995; Guillén et al. 1997; Bostrom et al. 2002). Los HAP también han contaminado los sistemas de agua dulce (Couch y Harchbarger 1985; Weeks et al. 1990). Los HAP son potencialmente cancerígenos (Shailaja y D'Silva 2003). Algunos HAP son considerados agentes causantes de cáncer de pulmón, de esófago, gástrico, colorrectal, vejiga, piel y próstata y cánceres de cuello uterino en los seres humanos y modelos animales (ATSDR 1995). Debido a su potencial cancerígeno y mutagénico, los HAP tienen, desde un punto de vista toxicológico, considerable interés (Armstrong et al. 2004). Los seres humanos están expuestos a los HAP a través de múltiples vías, incluyendo la inhalación de aire contaminado y la ingestión de alimentos que contienen hidrocarburos aromáticos policíclicos (Ramesh et al. 2004). Algunos autores han demostrado que los escapes de los motores de los vehículos son probablemente la fuente más importante de HAP actualmente detectada (Fang et al. 2004; Culotta et al. 2005). Una vez producidos, los HAP se pueden dispersar ampliamente a través del medio ambiente en el aire, agua, pueden acumularse en los suelos y sedimentos (Bartos et al. 2009; Danyi et al. 2009; Zhang et al. 2009). Después de entrar en el cuerpo humano, los HAP se someten a una serie de procesos de biotransformación. Durante el metabolismo de fase I, los HAP se oxidan por las enzimas del citocromo P450 para formar intermedios epóxidos altamente reactivos (Grover 1986). En las células mamarias, los HAP sufren activación metabólica hasta epóxidos diolicos que se enlazan covalentemente a macromoléculas celulares incluido el ADN, causando errores en la replicación del ADN y mutación (USEPA 2002; Yoon et al. 2007), que constituyen la etapa inicial de los procesos carcinogénicos (Janoszka et al. 2004). Los HAP pueden crear toxicidad en organismos, al interferir con la 
función de la membrana celular y los sistemas de acoplamiento de enzimas; los metabolitos de HAP se pueden unir al ADN que causa interrupciones bioquímicas y daño celular a los organismos (Kap et al. 2004; Gozgit et al. 2009; Jung et al. 2011). Muchos de los HAP individuales son citotóxicos, mutagénicos y potencialmente carcinogénicos para los seres humanos (WHO 1998). Los peces recolectados de aguas contaminadas por HAP presentan anormalidades externas y se piensa que estas están relacionadas con la inmunosupresión, incluyendo lesiones de las branquias, piel y aletas causadas por diferentes infecciones (Seeley y Week 1991). Sin embargo, es poco lo que se conoce sobre el mecanismo por el cual los HAP inducen inmunotoxicidad en los peces.

En recientes estudios en peces se ha investigado el mecanismo de la inmunotoxicidad inducida por los HAP (Faisal y Huggett 1993; Carlson et al. 2004; Reynaud y Deschaux 2005). Los mecanismos de defensa inmune adquirido de pescado son los mismos que los de los mamíferos (Zelikoff 1998). Las células inmunes que producen anticuerpos, se cree son análogas a los linfocitos B de mamíferos, se encuentran en el bazo y el riñón de los peces (Zelikoff 1998). Sin embargo, a diferencia de las cinco clases de inmonoglobulina (Ig) que se encuentran en los mamíferos, los anticuerpos producidos por los peces parecen estar restringidos a dos clases de Ig muy parecidas mammalian IgM e IgD (Zelikoff 1998; Srisapoome et al. 2004). Recientemente, un nuevo tipo de anticuerpo Ilamado IgT ha sido identificado en la trucha arco iris (Oncorhynchus mykiss) (Hansen et al. 2005). Los HAP pueden inducir un efecto inmunológico en la apoptosis de las células del sistema de los peces (Buchiel y Luster 2001; Weber y Janz 2001); Weyts et al.
(1997) han demostrado que la apoptosis como un mecanismo regulador inmune se conserva en el pescado que demuestra su importancia en el mantenimiento de la homeostasis inmunológica. En los mamíferos, una parte de la inmunotoxicidad puede estar mediado por la inducción de la muerte celular programada en los linfocitos (Hardin et al. 1992; Hinoshita et al. 1992; Yamaguchi et al. 1996). En los peces, un menor número de estudios se han realizado para definir el papel de la apoptosis en la inmunotóxicidad mediada por los HAP. In vivo la exposición a HAP inducida por la apoptosis en los eritrocitos de anguila (Nigro et al. 2002) y el bagre de canal (Ictalurus punctatus), que viven en agua contaminada por HAP, muestran un aumento de eritrocitos apoptósicos (Frenzilli et al. 2004).

El pescado es un producto de consumo masivo en la canasta familiar; perecedero, además es considerado una fuente sana de alimentación, que puede presentar niveles de toxicidad, debido a la influencia de la contaminación acuática y el daño alimentario que puede causar a largo, mediano y corto plazo. Como no se han encontrado estudios colombianos sobre la identificación de HAP en los alimentos, se hace necesario investigar en la identificación de HAP en la matriz alimentaria de productos de consumo masivo en el país y de esta manera, establecer elementos de seguimiento que permitan garantizar aspectos de seguridad alimentaria. El objetivo de la presente investigación fue adelantar una investigación preliminar sobre la presencia y naturaleza de hidrocarburos aromáticos policíclicos considerados como contaminantes prioritarios, en el músculo y piel de truchas arco iris provenientes de un sistema de cultivo intensivo ubicado en el municipio de 
Mutiscua (Departamento de Norte de Santander, Colombia).

\section{MATERIALES Y MÉTODOS}

Mutiscua está ubicada en el departamento de Norte de Santander, con las siguientes coordenadas geográficas: longitud Oeste $71^{\circ} 45^{\prime} 02^{\prime \prime}$, latitud Norte $07^{\circ} 18^{\prime} 07^{\prime \prime}$. Altitud 2600 m.s.n.m. y temperatura media de $14^{\circ} \mathrm{C}$. La trucha es la principal especie que se cría en el municipio y se ha convertido en una alternativa importante para la zona. Debido a las condiciones topográficas y climatológicas, y a la oferta hídrica se han consolidado diversas estaciones truchícolas; para la producción se cultiva la trucha Arcoíris, tipo Kam-Loop procedente de Estados Unidos. Mensualmente, se siembran 60.000 truchas con un período de cosecha de 10 meses.

Mutiscua se posiciona como uno de los principales comercializadores de trucha arcoíris en el mercado nacional y departamental con una producción de 25 toneladas al mes consolidándose como el principal productor en el departamento Norte de Santander.

Mutiscua está ubicada a dos km de la vía nacional que comunica a Pamplona con Bucaramanga, caracterizada por una alta influencia de vehículos pesados-tractomulas que funcionan con diésel y vehículos a gasolina. La trucha arco iris se adquirió en los estanques de la zona de Mutiscua.

Para obtener la muestra se aplicó muestreo no probabilístico con tamaño de muestra intencional, de tres peces por estanque, se seleccionaron tres estanques para un total de 18 ejemplares, el promedio de los ejemplares tenía un peso de 450 g y una longitud de 45 $\mathrm{cm}$.

\section{Extracción y concentración de la materia orgánica}

La materia orgánica presente en músculo y piel de los ejemplares se extrajo mediante ultrasonido; se utilizó el diclorometano como solvente de extracción. La piel y músculo de la trucha por separado (2 g), se colocaron en un vaso de precipitado con $50 \mathrm{~mL}$ del solvente durante una hora a una temperatura de $23{ }^{\circ} \mathrm{C}-24{ }^{\circ} \mathrm{C}$.

Una vez obtenido el extracto orgánico, se filtra y se concentra en un rota evaporador hasta aproximadamente $5 \mathrm{~mL}$ (extracto global). Posteriormente el extracto global se utilizó, para la determinación de Hidrocarburos Aromáticos Policíclicos (HAP) por Cromatografía de Gases.

Las muestras de HAP (músculo y piel de la trucha) se secaron con $\mathrm{Na}_{2} \mathrm{SO}_{4^{\prime}}$ con el fin de eliminar el agua residual y preparar la muestra para el análisis cromatográfico.

\section{Identificación de Hidrocarburos Aromáticos Policíclicos (HAP)}

Para identificar los Hidrocarburos Aromáticos Policíclicos (HAP), se utilizó un equipo de Cromatografía de Gases Agilent Technologies 6890A Plus Series II Hewlet-Packard Plus con detector FID (Flamelonization Detector). La columna utilizada fue Restek Rxi-17 Sil MS, $30 \mathrm{~m}$ de longitud, 0,25 mm de diámetro, 0,25 $\mu \mathrm{m}$ de diámetro interno (silarylene similar a 50\% phenyl $/ 50 \%$ dimethylpolysiloxane). Para la identificación de los HAP se utilizó el patrón de 16 hidrocarburos de Restek (catalogo \# 31841 EPA Method 8310 PAH Mixture). La identificación cualitativa de los HAP presentes en el extracto global se realizó de acuerdo a las siguientes condiciones: temperatura del 
inyector $250{ }^{\circ} \mathrm{C}$; detector FID a $320^{\circ} \mathrm{C}$ Mezcla (mL/min): aire $400-\mathrm{H}_{2} 30-\mathrm{N}_{2} 45$. Se inyectó $1 \mu \mathrm{L}$, modo splitless. Condiciones del horno: Temperatura inicial $65{ }^{\circ} \mathrm{C}$ por 0,5 min y se incrementa de la siguiente manera: $15{ }^{\circ} \mathrm{C} / \mathrm{min}$ hasta $200{ }^{\circ} \mathrm{C}, 4{ }^{\circ} \mathrm{C} / \mathrm{min}$ hasta $330{ }^{\circ} \mathrm{C}$ durante $15{ }^{\circ} \mathrm{C} / \mathrm{min}$. Gas de arrastre Helio, flujo $20 \mathrm{~mL} /$ min. Tiempo de análisis por muestra 53,33 min.

\section{RESULTADOS Y DISCUSIÓN}

Para la identificación de los diferentes HAP presentes en el extracto global tanto en piel como en músculo de truchas destinadas a consumo, se tomó como referencia el cromatógrama de la muestra patrón de 18 Hidrocarburos aromáticos policíclicos (EPA Method 8310 PAH Mix.).

En la figura 1se muestra el cromatógrama del estándar de los 18 HAP (Restek EPA method 8310 PAH Mix), que corresponden al:
1. Naftaleno

2. 1-Metilnaftaleno

3. 2-Metilnaftaleno

4. Acenaftileno

5. Acenafteno

6. Fluoreno

7. Fenantreno

8. Antraceno

9. Fluoranteno

10. Pireno

11. Benzo(a)antraceno

12. Criseno

13. Benzo(b)fluoranteno

14. Benzo(k) fluoranteno

15. Benzo(a) pireno

16. Indeno(1,2,3-cd) pireno

17. Dbenzo(a,h) antraceno

18. Benzo(ghi) perileno

En este caso el cromatógrama que se muestra en la figura 1 se obtuvo con la columna RESTEK Rxiß-17Sil MS.

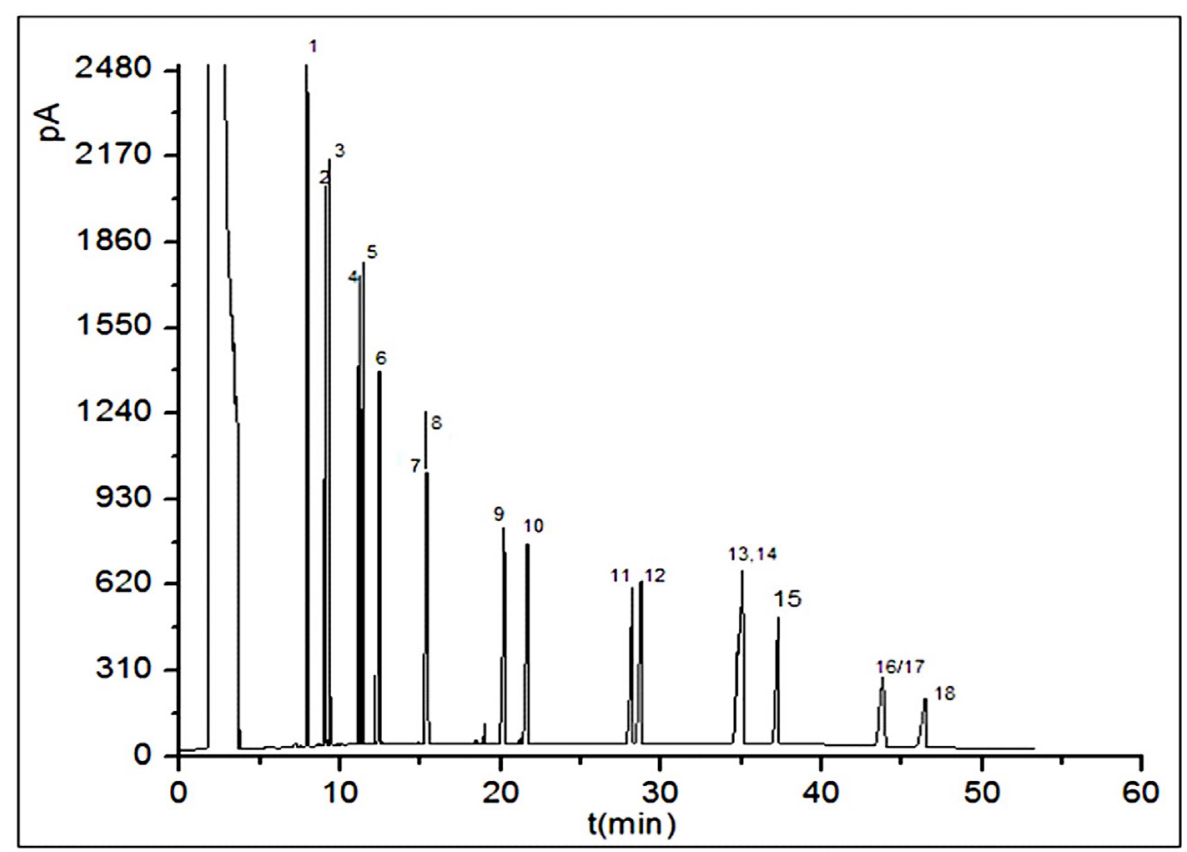

Figura 1. Cromatograma del patrón estándar de los 18 HAPs (Restek EPA method 8310 PAH Mix) 
En la figura 2 se presenta el perfil cromatográfico en el músculo de las truchas y se observa que en el tiempo de retención de los 35 min aparece la mezcla de dos HAP que corresponden a los Benzo (b), benzo(k) fluorantenos, los que están incluidos en la lista de los HAP8.

En la figura 3 se tiene el perfil cromatográfico que corresponde al análisis de la piel en los ejemplares; se observa que en el intervalo de 35 a 40 min, aparece el benzo (a) pireno, considerado como altamente carcinógeno por la IARC.
La contaminación del agua y el potencial resultante de bioacumulación de los contaminantes en los organismos acuáticos presenta un riesgo creciente para la vida silvestre y los seres humanos. Los hidrocarburos aromáticos policíclicos necesitan especial atención debido a su conocida toxicidad (Couillard, 2002) y que son potencialmente cancerígenos (Shailajay D'Silva, 2003); estos compuestos pueden inducir lesiones hepáticas, fisiológicas y trastornos bioquímicos en los peces. Se ha demostrado que en la trucha

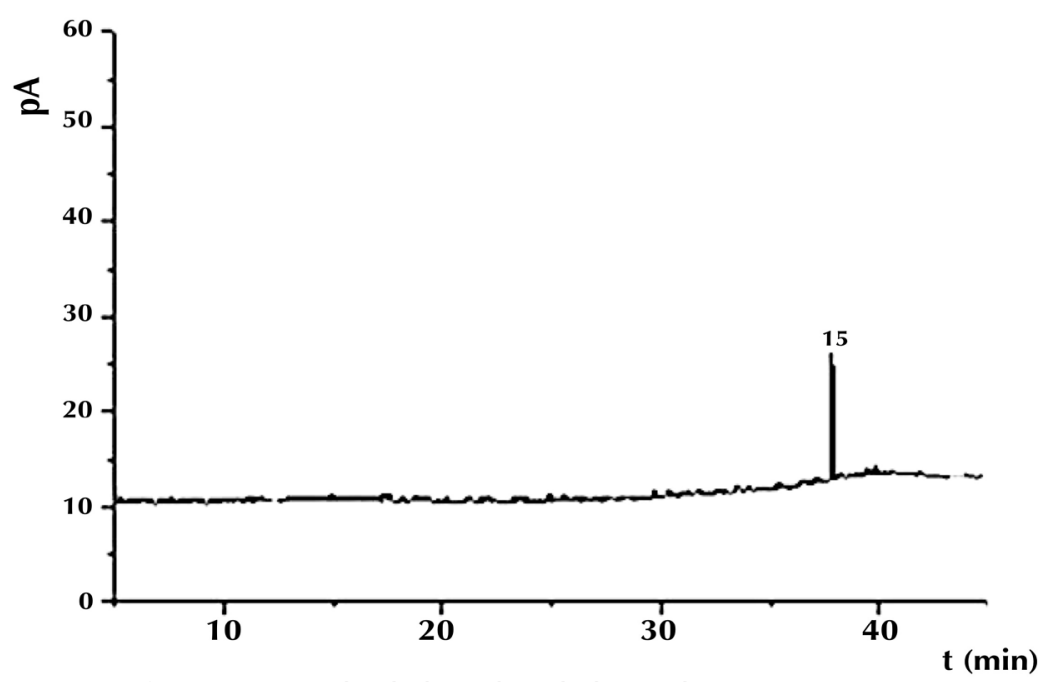

Figura 2. Perfil cromatográfico en músculo de la pulpa de la trucha arco iris

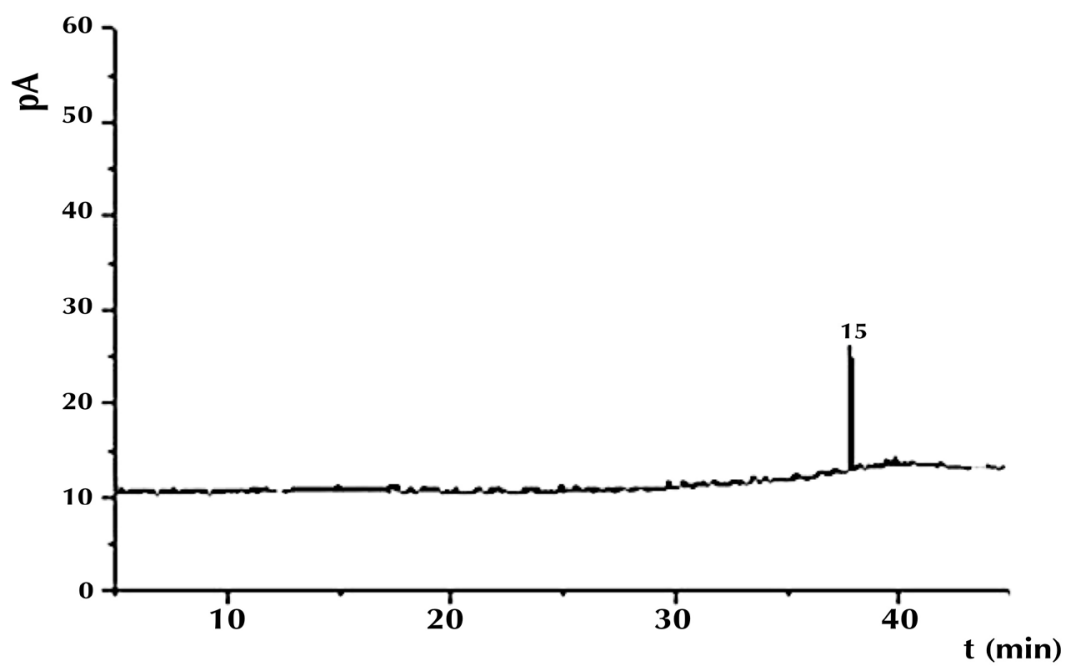

Figura 3. Perfil cromatográfico de la piel de la trucha arco iris 
expuesta a HAP, se bioacumulan aumentando los niveles de contaminantes en los tejidos con el tiempo (Hellou et al. 2002; Hellou y Leonard 2004). La Agencia de Protección Ambiental de EE.UU. (USEPA) recomienda el monitoreo de ciertos HAP conocidos como contaminantes prioritarios (Shibamoto 1998; USEPA 1986) y ha propuesto una selección de HAP que se encuentran frecuentemente en monitoreos de muestras ambientales a saber, Naftaleno ( $\mathrm{Na}$ ), Acenafteno (Ac), Acenaftileno (Ace), Fluoreno (F), Antraceno (A), Fenantreno $(\mathrm{Pa})$, Fluoranteno $(\mathrm{Fl})$, Pireno $(\mathrm{P})$, Benzo(a) antraceno (BaA), Criseno (Ch), Benzo(b) fluoranteno $(\mathrm{BbF})$, Benzo (k) fluoranteno $(\mathrm{BkF})$, Benzo(a)pireno (BaP), $\operatorname{dibenzo}(\mathrm{a}, \mathrm{h})$ antraceno (DhA), Benzo(g,h,i)perileno (BgP), Indeno(1,2,3-cd)pireno (IP) (USEPA 1984). El Benzo(a)pireno está clasificado en el grupo 1 como carcinógeno para humanos; la mezcla de Benzo(b)fluoranteno, Benzo(k)fluoranteno se clasifica en el grupo $2 \mathrm{~B}$ como posiblemente carcinogénicos para humanos (USEPA 2002; IARC 2004; IARC 2010).

De estos HAP conocidos como prioritarios, se recomienda el monitoreo de ocho HAP de elevado peso molecular (HAP8) como el BaA, Ch, BbF, BkF, BaP, IP, BgP, DhA (EC 2005).

Según la opinión del panel científico de contaminantes en la cadena alimentaria (EFSA 2008) el BaP no es el indicador más conveniente de la presencia de HAP en los alimentos y considera que la presencia de los HAP8 son los más indicados en los alimentos. El BaP es clasificado por la Agencia Internacional para la Investigación sobre el Cáncer (IARC) como carcinógeno para los humanos (IARC 2010), mientras que otros HAP se consideran probables y posibles carcinógenos en humanos (IARC
2002). El Benzo [a] pireno es probablemente el HAP carcinógeno más conocido y en muchos estudios de estimación de riesgos de cáncer humano, se utiliza a menudo como un sustituto de otros HAP cancerígenos. El análisis de HAP en peces es particularmente difícil debido a la baja concentración de los compuestos individuales, ya que generalmente la determinación o la presencia de estos son a nivel de trazas.

El creciente interés existente en la actualidad por la investigación en el campo de los compuestos orgánicos como los HAP, se debe a la identificación de efectos adversos de estos contaminantes sobre la salud y los ecosistemas. Es de anotar que los Hidrocarburos aromáticos policíclicos (BaP y la mezcla BbkF) detectados en la truchas, están considerados dentro del grupo de los HAP8 como contaminantes prioritarios y como indicadores posibles de la potencia carcinogénica de los HAP en los alimentos; provienen exclusivamente de la combustión de las fuentes móviles que circulan con diesel y gasolina.

Estos resultados nos indican la necesidad de establecer en Colombia un programa de vigilancia que incluya la determinación de las concentraciones de los HAP en los productos alimenticios, ya que los HAP encontrados, pueden ocasionar a largo plazo un riesgo para la salud humana.

\section{CONCLUSIONES}

De los HAP8 estudiados en músculo y piel de truchas cultivadas se encontraron los benzofluorantenos y el benzo(a)pireno clasificados como carcinógenos; se trata de contaminantes altamente peligrosos por presentar actividad mutagénica y genotóxica. 


\section{REFERENCIAS}

Armstrong, B., Hutchinson, E., Unwin, J. and Fletcher, T. 2004. Lung cáncer risk after exposure to polycyclic aromatic hydrocarbons: a review and meta-analysis. Environ. Health Perspect 112:970-978.

ATSDR. 1995. Toxicological Profile for Polycyclic Aromatic Hydrocarbons, Atlanta: Agency for Toxic Substances and Disease Registry. Available from: /http://www.atsdr.cdc.gov/ toxprofiles/tp69.htm

Bartos, T., Cupr, P., Klánová, J. and Holoubek, I. 2009. Which compounds contributed most to elevated airborne exposure and corresponding health risks in the Western Balkans? Environ Int 35:1066-71.

Bostrom, C., Gerde, P., Hanberg, A., Jernstrom, B., Johansson, C., Kyrklund, T., Rannug, A., Tornqvist, M., Victorin, K. and Westerholm, R. 2002. Cancer risk assessment, indicators, and guide lines for polycyclic aromatic hydrocarbons in the ambient air. Environ. Health Perspect 110 (Suppl. 3):451-488.

Buchiel, S. and Luster, M. 2001. Signaling by environment alpolycyclic aromatic hydrocarbons in human Iymphocytes. Clin. Immun 98:2-10.

Carlson, E., Li, Y. and Zelikoff, J. 2004. Benzo[a]pyrene-induced immuno- toxicity in Japanese medaka (Oryzias latipes): relationship between lymphoid CYP1A activity and humoral immune suppression. Toxicol. Appl. Pharmacol 201:40-52.

Couch, J. and Harchbarger, J. 1985. Effects of carcinogenic agents on aquatic animals: an environmental and experimental review. Environ. Carcinogenis 3:63-105.

Couillard, C. 2002. A microscale test to measure petroleum oil toxicity to mummichogembryos. Environmental Toxicology 17:195-202.

Culotta, L., Gianguzza, A. and Orecchio, S. 2005. Leaves of nerium oleander L. as bioaccumulators of polycyclic aromatic hydrocarbons $(\mathrm{PAH})$ in the air of Palermo (Italy). Extration. GCMS analysis, distribution, sources, Polycyclic Aromat. Compd 25:327344.

Danyi, S., Bose, F., Brasseur, C., Schneider, Y., Larondelle, Y. and Pussemier, L. 2009. Analysis of EU priority polycyclic aromatic hydrocarbons in food supplements using high performance liquid chromatography coupled to an ultraviolet, diode array or fluorescence detector. Anal Chem Acta 633:293-9.

EC (Commission Regulation), No 466/2001 of 4 February 2005. Off. J. Eur. UnionLO34(2005) 3-5. 
EFSA. European Food Safety Agency. 2008. Polycyclic Aromatic Hydrocarbons in Food. Scientific Opinion on the Panel on Contaminants in the Food Chain (Question No EFSA-Q-2007-136). EFSA J 724:1-114.

Faisal, M. and Huggett, R. 1993. Effects of aromatic hydrocarbons on the lymphocyte mitogenic responses in spot (Leiostomus xanthurus). Mar. Environ. Res 35:121-124.

Fang, G., Chang, C., Wu, Y., Fu, P., Yang, I. and Chen, M. 2004. Characterization, identification of ambient air and road dust polycyclic aromatic hydrocarbons in central Taiwan, Taichung, Sci. Total Environ 327:135-146.

Frenzilli, G., Scarcelli, V., Del Barga, I., Nigro, M., Forlin, L., Bolognesi, C. and Sturve, J. 2004. DNA damage in eelpout (Zoarces viviparus) from Goteborg harbour. Mutat. Res 552:187-195.

Guillen, M., Sopelana, P. and Partearroyo, M. 1997. Food as a source of polycyclic aromatic carcinogens. Rev. Environ. Health 12:133-146.

\section{Gozgit, J., Nestor, K., Fasco, M., Pentecost,} B. and Arcaro, V. 2009. Differential action of polycyclic aromatic hydrocarbons on endogenous estrogen-responsive genes andona transfected estrogen-responsive reporter in MCF-7cells. Toxicol.
Appl. Pharmacol 196:58-67.

Grover, P. 1986. Path ways involved in the metabolism and activation of polycyclic hydrocarbons. Xenobiotica 16:915-931.

Hansen, J., Landis, E. and Phillips, R. 2005. Discovery of a unique Ig heavy-chain isotype $(\lg \mathrm{T})$ in rainbow trout: implications for a distinctive $B$ cell developmental pathway in teleost fish. Proc. Natl. Acad. Sci. U.S.A.102:6919-6924.

Hardin, J., Hinoshita, F. and Sherr, D., 1992. Mechanisms by which benzo[a]pyrene, an environmental carcinogen, suppresses B cell lymphopoiesis. Toxicol. Appl. Pharmacol 117:155-164.

Hellou, J. and Leonard, J. 2004. Polycyclic aromatic hydrocarbons bioaccumulation and biotransformation products in trout exposed through food pellets. International Journal of Polycyclic Aromatic Compounds 24:1-15.

Hellou, J., Leonard, J. and Antsey, C. 2002. Dietary exposure of finfish to aromatic contaminants and tissue distribution. Archives of Environmental Contamination and Toxicology 42:470-476.

Hinoshita, F., Hardin, J. and Sherr, D. 1992. Fluoranthene induces programmed cell death and alters growth 
of immature B cell populations in bone marrow cultures. Toxicology 73:203-218.

International Agency for Research on Cancer (IARC). 2010. Some nonheterocyclic polycyclic aromatic hydrocarbons and some related exposures. IARC Monographs on the Evaluation of Carcinogenic Risks to Humans 92:773.

International Agency for Research of Cancer (IARC). 2004. Overallevaluationsofcarcinogenicity: anupdating of IARC monographs. Int Agency Res Cancer.7

International Agency for Research on Cancer (IARC). 2002. Some traditional herbal medicines, some mycotoxins, naphthalene and styrene. IARC Monographs on the Evaluation of Carcinogenic Risks to Humans 82:367.

International Agency for Research on Cancer (IARC). 1983. Polyciclic Aromatic Compounds. Part I. Chemicaland, environment and Experimental Data, IARC. Monographs on the Evaluation of carcinogen Risk of chemicals to umans. vol.32. Available from:/ http://monographs.iarc.fr/ENG/ Monographs/vol32/volume32.pdfS. (last accessed 14.06.07).

Janoszka, B., Warzecha, L., Blaszczyk, U. and Bodzek, D. 2004. Organic compounds formed in thermally treated high-protein food. Part I: polycyclic aromatic hydrocarbons. Acta Chromatica 14:115-28.

Jung, D., Matson, C., Collins, L., Laban, G., Stapleton, H., Bickham, J., Swenberg, J.. and Di Giulio, R. 2011. Genotoxicity in Atlantickilli fish (Fundulus heteroclitus) from a PAH-contaminated Superfund site on the Elizabeth River, Virginia. Ecotoxicology 20:1890-1899.

Kap, N., Do, L., Cha, J., Joo, WA., Lee, E and Chan, K. 2004. Protein biomarkers in the plasma of workers occupationally exposed to polycyclic aromatic hydrocarbons, Proteomics 4:3505-3513.

Mudzinski, SP. 1993. Effects of benzo[a]pyrene on concanavalin A-stimulated human peripheral blood mononuclear cells in vitro: inhibition of proliferation but no effect on parameters related to the G1 phase of the cell cycle. Toxicol. Appl. Pharmacol 119:166-174.

Nigro, M., Frenzilli, G., Scarcelli, V., Gorbi, S. and Regoli, F. 2002. Induction of DNA strand breakage and apoptosis in the eel Anguilla anguilla. Mar. Environ. Res. 54:517-520.

Ramesh, A., Walker, S., Hood, D., Guillen, M., Schneider, K. and Weyand, E. 2004. Bioavailability and risk assessment of orally ingested 
polycyclic aromatic hydrocarbons. Int. J. Toxicol 23:301-333.

Reynaud, S. and Deschaux, P. 2005. The effects of 3-methylcholanthrene on lymphocyte proliferation in the common carp (Cyprinus carpio L.). Tox-icology 211:156-164.

Seeley, K. and Week, B. 1991. Altered phagocytic activity of macrophages in oyster toadfish from a highly polluted estuary. J. Aquat. Animal Health 3:224-227.

Shailaja, M. and D'Silva, C. 2003. Evaluation of impact of $\mathrm{PAH}$ on atropical fish. Oreochromis mossambicus using multiple biomarkers. Chemosphere 53:835841.

Shibamoto, T. 1998. Chromatographic Analysis of Environmental and Food Toxicants. Marcel Dekker, INC., New York

Srisapoome, P., Ohira, T., Hirono, I. and Aoki, T. 2004. Genes of the constant regions of functional immunoglobulin heavy chain of Japanese flounder, Paralichthys olivaceus. Immunogenetics 56:292300.

USEPA. United States Environmental Protection Agency. 2002. Polycyclic Organic Matter. Environmental Protection Agency, Washington, DC.
Available at: http://www.epa.gov/ttn/ atw/hlthef/polycycl.html.

USEPA. United States Environmental Protection Agency. 1986. Guidelines for Carcinogen Risk Assessment. Federal Register, 51(185), pp. $33992-34003$. E P A / 630 / R 00/004,Washington, DC.

\section{USEPA. United States Environmental Protection Agency. 1984. Polynuclear Hydrocarbons, vol 10.US-EPA, Washington,DC.}

Weber, L. and Janz, D. 2001. Effect of _-dimethylbenz[a] anthraceneon apoptosis and HSP70 expression in juvenilecannel catifish (Ictalurus punctatus) ovary. Aquat. Toxicol 54:39-50.

Weeks, B., Warinner, J., Mathews, E. and Wishkovsky, A. 1990. Effects of toxicant on certain functions of the Iymphoreticular system of fish.Patho. Mar. Sci 32:369-374.

Weyts, F., Verburg, B., Flik, G., Lambert, J. and Wende, S. 1997. Conservation of apoptosis as an immune regula-tory mechanism: effects of cortisol and cortisone on carp lymphocytes. Brain Behav. Immun 11:95-105.

\section{WHO. World Health Organization.}

1998. Environmental Health Criteria 202: Selected Non-Heterocyclic Polycyclic Aromatic Hydrocarbons. 
Yamaguchi, K., Near, R., Shneider, A., Cui, H., Ju, ST. and Sherr, D. 1996. Fluoranthene-induced apoptosis in murine T cell hybridomas is independent of the aromatic hydrocarbon receptor. Toxicol. Appl. Pharmacol 139:144-152.

Yoon, E., Park, K., Lee, H., Yang, J and Lee, C. 2007. Estimation of excess cancer risk on time-weighted lifetime average daily intake of PAHs from food ingestion. Hum Ecol Risk Assess 13:669-80.
Zelikoff, J. 1998. Biomarkers of immunotoxicity in fish and other non-mammalian sentinel species: predictive value for mammals? Toxicology 129:63-71

Zhang, P., Song, J. and Yuan H. 2009. Persistent organic pollutant residues in the sediments and mollusks from the Bohai Sea coastal areas, North China: an overview. Environ Int 35:632-46.

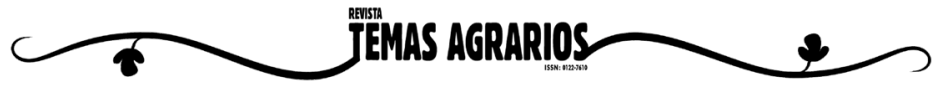

